\title{
Rehabilitación de viviendas con criterios de accesibilidad en España. Respuestas inclusivas ante la diversidad humana y social
}

Using accessibility criteria for dwelling rehabilitation in Spain. Inclusive responses for human and social diversity.

Yolanda María de la Fuente R. - Jesús Hernández G.

\section{Filiación}

Universidad de Jaén - Fundación Once

E mail: ymfuente@ujaen.es - jhernandez@fundaciononce.es

\section{Resumen}

Las Viviendas de Promoción Pública, que tienen como misión satisfacer la necesidad de vivienda de ciudadanos con rentas bajas, pierden este fin cuando las mismas no dan respuesta a las necesidades de la población que atienden, partiendo de la premisa de que las mismas fueron diseñadas al margen de criterios de accesibilidad y de lo que se entiende en la actualidad como Design for all.

Cuando la población que hacía uso de las mismas era joven e independiente respondía al derecho a la vivienda, pero ahora esa población es una población envejecida, con recursos reducidos y espoleada por la crisis económica; nos encontramos así con un sector de población doblemente excluido, son mayores, dependientes y con bajos recursos, si a todo esto unimos a las personas con discapacidad tanto de origen como sobrevenida, forman parte de una población invalidada por el entorno hostil de la vivienda en la que residen, convirtiéndose en obstáculos imposibles de remover por falta de recursos y de estrategia adaptada para intervenir en este sector. El objetivo de este artículo pretende demostrar la necesidad de establecer un protocolo de información y actuación en materia de accesibilidad para llevar a cabo la adaptación de las viviendas atendiendo a la individualidad de sus moradores. Para ello se ha llevado a cabo una revisión profunda de la normativa especializada en la materia, argumentando las oportunidades que ofrece la misma y que hasta la fecha no han sido desarrolladas o simplemente acatadas.

\section{Palabras Claves}

Accesibilidad; Rehabilitación; Inclusión; Diseño para Todos

\begin{abstract}
The main purpose of Public Housing, that is, to satisfy the needs of citizens with low incomes, is lost when the design and construction standards of public housing projects do not take into consideration accessibility criteria, also known as Design for All.

As the population that occupies public housing projects gets older, and their needs become more specific and harder to fulfill, resources stop flowing in, putting them in a double exclusion situation: on the one hand, they depend on others to be able to execute simple day to day chores in a hostile housing environment, and on the other, they don't have resources to transform that poorly planned environment. Therefore, the main goal of this article is to stress the need of implementing information and action protocols, regarding accessibility, in order to be able to adapt existing public housing to the specific requirements of their dwellers. The research team has performed an in depth critical review of the existing regulations specifically dedicated to accessibility, finding an array of opportunities that have not been used or developed.
\end{abstract}




\section{URBANI SMO}

ISSN 0717-5051

http://revistas.uchile.cl/index.php/RU/index
Revista de Urbanismo $\mathrm{N}^{\circ} 28$ - Junio de 2013

Departamento de Urbanismo - FAU - Universidad de Chile

\section{Key words}

Accessibility; rehabilitation; inclusion; Design for all.

\section{Sumario}

Introducción

1. Marco Teórico: El cuerpo jurídico español en materia de accesibilidad

2. Metodología: Programa Individualizado de Accesibilidad

Resultados y Conclusiones: Efectos colaterales positivos de las intervenciones rehabilitadoras

Bibliografía

\section{I ntroducción}

Para hablar de accesibilidad universal, hay que partir del concepto de diseño universal, que según la Convención de la ONU sobre los Derechos de las Personas con Discapacidad es "el diseño de productos, entornos, programas y servicios que puedan utilizar todas las personas, en la mayor medida posible, sin necesidad de adaptación ni diseño especializado". No obstante, la Convención Internacional sobre los Derechos de las Personas con Discapacidad aclara que el diseño universal "no excluirá las ayudas técnicas, para grupos particulares de personas con discapacidad cuando se necesiten".

Como se indica en el Libro Verde de la Accesibilidad ${ }^{1}$, el término accesibilidad, proviene de acceso, acción de llegar y acercarse, o bien entrada o paso. Aplicado al uso del espacio o de los objetos y tecnologías, y especialmente con relación a ciertos colectivos de población con dificultades funcionales, la palabra adquiere un matiz menos neutro, más expresivo de los beneficios que se derivan de la interacción con el entorno o con otras personas.

Por otro lado, según el Concepto Europeo de Accesibilidad, es una característica básica del entorno construido, la condición que posibilita llegar, entrar, salir y utilizar las casas, las tiendas, los teatros, los parques y los lugares de trabajo, permitiendo a las personas participar en las actividades sociales y económicas para las que estas se han concebido.

De esto se desprende que, cuando exista una falta de accesibilidad, por ejemplo, con barreras físicas en su vivienda, esa persona sufrirá algún grado de marginación y, lógicamente, una pérdida de calidad de vida respecto a los demás que no la sufran. El propio Concepto Europeo de Accesibilidad $^{2}$ también especifica que el término accesibilidad puede entenderse en relación con tres formas básicas de actividad humana: movilidad, comunicación y comprensión.

Como señala Juncá:

“La accesibilidad universal es una actitud ante la vida, ese difícil arte, y artesanía a la vez, de aplicar criterios sólidos en el diseño urbano o arquitectónico que se sustenten en proporcionar el bienestar, la comodidad y la seguridad de quienes lo utilicen"3

\footnotetext{
1 IMSERSO, 2002.

2 IMSERSO, 1996.

3 Juncà, 2010. p. 7.
} 


\section{URBANI SMO}

ISSN 0717-5051

Revista de Urbanismo $\mathrm{N}^{\circ} 28$ - Junio de 2013

http://revistas.uchile.cl/index.php/RU/index

Departamento de Urbanismo - FAU - Universidad de Chile

Todos, según sean nuestras capacidades funcionales o mentales, tropezamos con barreras en nuestra capacidad de movimiento, nuestras comunicaciones o fuentes de información, y en nuestro alcance de comprensión de mensajes, instrucciones, instrumentos o sistemas. Por lo tanto, los entornos, productos y servicios accesibles mejoran la calidad de vida de todas las personas independientemente de cuáles sean sus capacidades funcionales. Obviamente para las personas con diversidad funcional, la accesibilidad es una necesidad básica para poder ejercer sus derechos de participación ciudadana en igualdad de condiciones que el resto de sus congéneres. Es por ello que la lucha por la obtención de entornos, productos y servicios accesibles ha surgido desde el movimiento asociativo de personas con discapacidad pero que sin duda alguna se ha convertido en un derecho fundamental de toda la ciudadanía. Según el I Plan Nacional de Accesibilidad aprobado por el Gobierno de España en el año 2003 el $40 \%$ de la población se beneficia directamente de la accesibilidad ${ }^{4}$, ya que como señalaba el European Institute of Design and Disability "el buen diseño capacita, el mal diseño discapacita"5

Si atendemos a la definición actual de accesibilidad, la misma considera la variedad de entornos y situaciones en que se debe hacer efectiva la participación en igualdad de condiciones de todas las personas, así como la diversidad de necesidades de éstas:

“Accesibilidad es el conjunto de características de que debe disponer un entorno, producto o servicio para ser utilizable en condiciones de confort, seguridad e igualdad por todas las personas y, en particular, por aquellas que tienen alguna discapacidad".

Estas características son referidas, en primer lugar al diseño de los entornos (edificios, espacios públicos, vehículos), productos (ergonomía, diseño industrial accesible) o servicios (telecomunicaciones, información, ocio.....); pero también a la forma de gestionarlos y a su correcto mantenimiento ${ }^{6}$.

Con esta realidad social definida con diversos conceptos, que han ido adaptándose unas veces a entorno, otras a productos y servicios; se ha hecho necesario la creación de un cuerpo jurídico suficiente que obligara tanto a administraciones públicas como al sector privado a introducir los criterios de diseño para todos y por tanto conseguir la accesibilidad en su mayor extensión posible, terminado así con la vacatio legis existente en la materia. Aunque a priori, parece evidente que la consecución de un marco legislativo es suficiente para que la accesibilidad universal se incorpore de forma natural a entornos productos y servicios, la realidad puede ser muy diferente.

Por todo ello, en este artículo analizaremos si el cuerpo jurídico y normativo español en materia de accesibilidad es suficiente y si su aplicación práctica está resultando realmente efectiva o es necesario, como aquí se propone, introducir de manera transversal el carácter individualizado en la intervención rehabilitadora, llevando a cabo un design thinking junto con el usuario, o lo que es lo mismo, realizar el camino inverso y situar a los destinatarios en el centro de la respuesta.

\section{Marco Teórico: El cuerpo jurídico español en materia de accesibilidad}

Se piensa habitualmente en la dependencia como un fenómeno que refleja la pérdida de autonomía personal cuya principal consecuencia es la necesidad por parte de la persona mayor o con discapacidad de ser ayudada.

${ }^{4}$ IMSERSO, 2003.

${ }^{5}$ EIDD, 2004.

${ }^{6}$ ACCEPLAN, 2003. 


\section{URBANI SMO}

Además, la pérdida de autonomía personal es definida, casi con exclusividad, en términos físicos o biológicos explicada como una consecuencia natural del paso de los años. Dejando de lado esta concepción unidimensional de la dependencia, es mejor pensar en la dependencia como un concepto de naturaleza compleja, con diversas caras, en el que influyen variables biológicas, psicológicas y sociales ${ }^{7}$.

En el tratamiento que se ha dispensado a las personas con discapacidad, es posible distinguir tres modelos, y que si bien coexisten en alguna medida en el presente, pueden ser situados en diferentes contextos históricos. Estos son: el modelo de prescindencia, el modelo rehabilitador y el modelo social.

Desde el modelo de prescindencia se considera que las causas que dan origen a la discapacidad son religiosas, y que las personas con discapacidad no tienen nada que aportar a la sociedad. El segundo modelo identificado es el denominado rehabilitador. En cuanto a las causas que dan origen a la discapacidad, se considera que son científicas: una limitación física, psíquica, mental o sensorial individual de la persona. Bajo este paradigma podría decirse entonces, que la vida de una persona con discapacidad se considera menos valiosa que la del resto de personas, aunque esa suposición puede ser revertida en el caso de que la persona sea rehabilitada. A pesar de representar un avance respecto del modelo de prescindencia, el modelo rehabilitador es criticado en cuanto a la filosofía que lo justifica, en lo que atañe a la consideración de la persona con discapacidad como un ser dependiente, inferior, que necesita ser rehabilitado a los efectos de recuperar su dignidad - una dignidad que se entiende lesionada o perdida-. Un tercer modelo que puede ser identificado es el denominado social. Desde este paradigma se considera que las causas que dan origen a la discapacidad no son religiosas ni científicas, sino que son preponderantemente sociales. Se parte de la premisa de que la discapacidad es una construcción y un modo de opresión social, y el resultado de una sociedad que no considera ni tiene presente a las personas con discapacidad. Así, se entiende que no son las limitaciones individuales las raíces del problema, sino las limitaciones de la sociedad para asegurar adecuadamente que las necesidades de todas las personas -incluyendo quienes tengan una discapacidad-sean tenidas en cuenta dentro de la organización social ${ }^{8}$.

En este sentido, Oliver ${ }^{9}$ plantea que el significado de discapacidad más que comprendido está distorsionado por las definiciones oficiales derivadas del paradigma de la rehabilitación como son las utilizadas por la Organización Mundial de la Salud. Esas definiciones y concepciones consideran a las personas con discapacidad como objetos pasivos de intervención, tratamiento y rehabilitación, generando consecuencias opresivas para las personas al reducir la discapacidad a un estado estático y violar sus componentes experienciales y situacionales. Oliver plantea como alternativa centrarse en las causas y dimensiones sociales de la discapacidad,

"una teoría social de la discapacidad debe estar localizada dentro de la experiencia de las propias personas con discapacidad y sus intentos, no solo para redefinir la discapacidad sino también para construir un movimiento político entre ellos mismos y desarrollar servicios proporcionados con sus propias necesidades autodefinidas"10.

\footnotetext{
7 Montorio, 2007.

8 Palacios, 2008.

9 Oliver, 1990.

${ }^{10}$ Oliver, 1990. p.11.
} 


\section{URBANI SMO}

El modelo social de la discapacidad ${ }^{11}$, sugiere que hay un lugar para la sensibilidad a la experiencia individual de la interacción entre la discapacidad y el deterioro. Una persona en riesgo de exclusión, marcada ésta por la situación económica, tiene menos oportunidades que una persona con discapacidad para acceder a los ajustes razonables que puedan minimizar el impacto de su discapacidad.

El logro de la claridad y la coherencia de una conceptualización social, relacional de la discapacidad en los estudios sobre discapacidad sería necesario como señala Thomas, iluminar la verdadera brecha entre este y la sociología médica, una brecha asociada con el hecho de que, hasta la fecha, médicos sociólogos no han estado dispuestos a reconocer que la discapacidad se asocia con la opresión social o de exclusión social sistemática, se han adoptado posturas más comedidas de que hay una cierta "desventaja social" que participan en deshabilitar la vida de las personas ${ }^{12}$ o lo que es lo mismo, los válidos invalidados por la coyuntura, sea esta entornos hostiles, físicos, virtuales, sociales, económicos, etc.

En el modelo social o universal, se plantea la discapacidad como un hecho universal en el que toda la población está en situación de riesgo. En el principio de universalización las iniciativas políticas deben dirigirse a toda la población, ya que todos están en riesgo de ser discapacitados, siendo la discapacidad y la capacidad los extremos de un continuo. Este modelo asume que la discapacidad no es un atributo que diferencia una parte de la población de otra, sino que es una característica intrínseca de la condición humana. La visión universal de la discapacidad supera los elementos de estigmatización y discriminación que la caracterizaban, pero sobre todo se está promoviendo en los países más avanzados donde no se ha establecido la diferenciación población discapacitada y no discapacitada ${ }^{13}$.

La legislación española ha ido introduciendo las premisas del modelo social o universal desde la aprobación de su Carta Magna en el año 1978, ya que en su artículo 9 exigía a los poderes públicos garantizar el máximo bienestar de vida a todos los ciudadanos, facilitando a su vez su participación en la vida política, económica, cultural y social. Asimismo, la Constitución, en su artículo 49, preconiza una política de previsión e integración de las personas con discapacidad.

Por otra parte, el artículo 148.1.3으 y 20으 del Texto Constitucional atribuye a las Comunidades Autónomas la competencia en materia de ordenación del territorio, urbanismo, vivienda y en materia de asistencia social. En virtud de tales competencias casi todas ellas han establecido normativa que garantiza, en mayor o menor medida, la accesibilidad en las ciudades, las edificaciones, el transporte y la comunicación, siendo la normativa básica al respecto la siguiente:

- Ley 20/1997, de 4 de diciembre, para la Promoción de la Accesibilidad del País Vasco

- Ley 20/1991, de 25 de noviembre, de promoción a la accesibilidad y supresión de barreras arquitectónicas de Cataluña, desarrollada por Decreto 135/1995, de 24 de marzo

- Ley 8/1997, de 20 de agosto, de accesibilidad y supresión de barreras en la Comunidad Autónoma de Galicia, desarrollada por Decreto 35/2000, de 28 de enero

${ }^{11}$ Shakespeare 2006.

12 Thomas, 2004.

13 INSTITUTO ARAGONÉS DE EMPLEO, 2005. 
- Decreto 72/1992, de 5 de mayo, por el que se aprueban las normas técnicas para la accesibilidad y la eliminación de barreras Arquitectónicas, Urbanísticas y en el Transporte en Andalucía

- Ley 5/1995, de 6 de abril, de promoción de la accesibilidad y supresión de barreras de Asturias, desarrollada por Decreto 37/2003, de 22 de mayo

- Ley 3/1996, de 24 de septiembre, sobre accesibilidad y supresión de barreras arquitectónicas, urbanísticas y de la comunicación de Cantabria

- Ley 5/1994, de 19 de julio, de supresión de barreras arquitectónicas y promoción de la accesibilidad de La Rioja, desarrollada por Decreto 19/2000, de 28 de abril

- Ley 5/1995, de 7 de abril, de condiciones de habitabilidad en edificios de viviendas y promoción de la accesibilidad general de la Región de Murcia, que, hasta la aprobación de su propio Reglamento, ha de entenderse desarrollada por Decreto 39/1987, de 4 de junio

- Ley 1/1998, de 5 de mayo, de accesibilidad y supresión de barreras arquitectónicas, urbanísticas y de la comunicación de la Comunidad Valenciana, desarrollada por Decreto 39/2004, de 5 de marzo

- Ley 3/1997, de 7 de abril, de Promoción de la Accesibilidad y Supresión de Barreras Arquitectónicas, Urbanísticas, de Transportes y de la Comunicación de Aragón, desarrollada por Decreto 19/1999, de 9 de febrero

- Ley 1/1994, de 24 de mayo, de accesibilidad y eliminación de barreras en Castilla-La Mancha, desarrollada por Decreto 158/1997, de 2 de diciembre

- Ley 8/1995, de 6 de abril, de accesibilidad y supresión de barreras físicas y de la comunicación de Canarias, desarrollada por Decreto 227/1997, de 18 de septiembre

- Ley 8/1997, de 18 de junio, de Promoción de la Accesibilidad en Extremadura, desarrollada por Decreto 8/2003, de 28 de enero

- Ley 3/1993, de 4 de mayo, para la mejora de la accesibilidad y de la supresión de las barreras arquitectónicas de las Islas Baleares, desarrollada por Decreto 20/2003, de 28 de febrero

- Ley 8/1993, de 22 de junio, de promoción de la accesibilidad y supresión de barreras arquitectónicas de la Comunidad de Madrid, desarrollada por Decreto 13/2007, de 15 de marzo

- Ley 3/1998, de 24 de junio, de Accesibilidad y Supresión de Barreras de Castilla y León, desarrollada por Decreto 217/2001, de 30 de agosto

- Ley Foral 4/1988, de 11 de julio, sobre barreras físicas y sensoriales de Navarra

Aun habiéndose constatado la competencia autonómica en materia de accesibilidad de las diferentes comunidades autónomas, es necesario destacar que desde la Administración Central se han ido aprobando normativas nacionales que de manera transversal y a veces con carácter específico, han ido perfilando la respuesta de la administración a diferentes colectivos, fundamentalmente el relativo al de las personas con discapacidad y posteriormente con el reconocimiento de un nuevo derecho subjetivo, el derecho a la atención a la dependencia, aunque como señala De Asis la proclamación de ese nuevo derecho de ciudadanía, podría 


\section{URBANI SMO}

ISSN 0717-5051

http://revistas.uchile.cl/index.php/RU/index
Revista de Urbanismo $N^{\circ} 28$ - Junio de 2013

Departamento de Urbanismo - FAU - Universidad de Chile

haberse visto acompañada de la proclamación de otro derecho de ciudadanía: el derecho a la accesibilidad universal ${ }^{14}$.

Pero antes de llegar a esta última norma, Ley 39/2006, de 14 de diciembre, de Promoción de la Autonomía Personal y Atención a las personas en situación de dependencia, comenzaremos por el primer texto legal español que introdujo el concepto de eliminación de barreras adoptando un nuevo planteamiento general de cara a la discapacidad basado en la supresión de las barreras a la plena participación en la sociedad, en lugar de «abordar el problema a escala individual», como defendía el planteamiento del «modelo médico» de la discapacidad, fue la Ley 13/1982 de 7 de Abril, de Integración Social de los Minusválidos. El tratamiento que esta Ley dio a la accesibilidad fue muy superficial dedicando a ello tan sólo la Sección 1a de su Título IX. Los entornos que aparecen en este texto como objetivos de mejora de la accesibilidad son básicamente entornos urbanos. Lo que si estableció esta ley fue la responsabilidad de las Comunidades Autónomas para desarrollar su propia legislación en materia de accesibilidad.

El siguiente texto legal que se aprobó en relación a la eliminación de barreras fue el Real Decreto 556/1989, de 19 de mayo, por el que se arbitran medidas mínimas sobre accesibilidad en los edificios.

Fue a finales de los años 80 y durante los años 90 cuando cada una de las Comunidades Autónomas ejerció su competencia aprobando sus propias leyes de eliminación de barreras arquitectónicas, urbanísticas, del transporte y la comunicación. Estas leyes se centraron especialmente en atender las necesidades de las personas con limitaciones en sus capacidades funcionales motoras, sin embargo se obviaron las necesidades de la ciudadanía con dificultades sensoriales, mentales o intelectuales.

A pesar del esfuerzo realizado tanto por el Gobierno español como por las Comunidades Autónomas por regular la accesibilidad arquitectónica, urbanística y del transporte, estas leyes nacieron sin régimen sancionador.

Esto significó que cualquier infracción cometida sobre estas leyes quedó impune produciéndose un incumplimiento permanente de las mismas. Esto quedó patente en el I Plan Nacional de Accesibilidad, aprobado el 5 de julio del 2003 y vigente durante el período 2004-2012 donde señalaba que

"Los trabajos de campo realizados con motivo del Plan muestran que las actuaciones prácticas en materia de accesibilidad suelen ser irregulares y descoordinadas, ofreciendo en balance un resultado insuficiente"15.

A grandes rasgos este Plan se constituyó como el documento rector para la promoción de la accesibilidad en España durante su periodo de vigencia. Asimismo estableció unos objetivos y una metodología transversal que son adecuados para desarrollar el lema del Plan: "Por un nuevo paradigma, el Diseño para Todos, hacia la plena igualdad de oportunidades". Es en este documento donde el concepto "eliminación de barreras" se sustituye por el concepto "diseño para todos" realizándose una revisión terminológica de modo que se eliminan términos negativos como eliminar o barreras adoptándose una terminología mucho más positiva e inclusiva. Sin embargo, según se pone de manifiesto en el manual La transversalidad de género en las políticas públicas de discapacidad ${ }^{16}$, este término incurre en el uso de terminología

\footnotetext{
14 De Asis, 2007.

15 IMSERSO, 2003.

${ }^{16}$ CERMI, 2012.
} 


\section{URBANI SMO}

ISSN 0717-5051

http://revistas.uchile.cl/index.php/RU/index
Revista de Urbanismo $N^{\circ} 28$ - Junio de 2013

Departamento de Urbanismo - FAU - Universidad de Chile

sexista. Este término proviene de la traducción del inglés Design for All por ello se propone como término más correcto Diseño para todas las personas.

Según el Plan, Diseño para Todos es la actividad por la que se concibe o proyecta, desde el origen y siempre que ello sea posible, entornos, procesos, bienes, productos, servicios, objetos, instrumentos, dispositivos o herramientas, de tal forma que puedan ser utilizados por todas las personas, en la mayor extensión posible. De esta forma, ambos conceptos, Igualdad de Oportunidades y Diseño para Todos, que son las dos grandes ideas que movilizan la lucha por la accesibilidad en los últimos años, aparecen conjuntamente como lema del Plan.

Este concepto plantea una actitud proactiva de superación del estigma de la diferencia con el que tradicionalmente han sido tratadas las personas con discapacidad. Además, enfatiza la atención a la diversidad de las capacidades funcionales del individuo teniéndolas presentes en los procesos de diseño de entornos, productos y servicios. La inclusión del diseño para todos de forma transversal en los procesos de diseño, redacción de proyectos y planificación es clave para que el resultado para que el resultado de esos procesos ya sean productos, entornos o servicios sean verdaderamente utilizables por todos ${ }^{17}$.

Siguiendo esta premisa, se aprobó la Ley 51/2003, de 2 de diciembre, de Igualdad de Oportunidades, no Discriminación y Accesibilidad Universal de las Personas con Discapacidad (LIONDAU). Esta Ley tiene por objeto establecer medidas para garantizar y hacer efectivo el derecho a la igualdad de oportunidades de las personas con discapacidad.

A pesar de la obligación legal de desarrollar la LIONDAU en el periodo de dos años para su aprobación no es hasta 2007 cuando se producen los nuevos desarrollos legislativos a través de los siguientes Reales Decretos:

- RD 366/2007, de 16 de marzo, por el que se establecen las condiciones de accesibilidad y no discriminación de las personas con discapacidad en sus relaciones con la Administración General del Estado

- RD 505/2007, de 20 de abril, por el que se aprueban las condiciones de accesibilidad y no discriminación de las personas con discapacidad para el acceso y utilización de los espacios públicos urbanizados y edificaciones

- RD 1494/2007, de 12 de noviembre, por el que se aprueba el Reglamento sobre las condiciones básicas para el acceso de las personas con discapacidad a las tecnologías, productos y servicios relacionados con la sociedad información y medios de comunicación social

- RD 1544/2007, de 23 de noviembre, por el que se regulan las condiciones básicas de accesibilidad y no discriminación para el acceso y utilización de los modos de transporte para personas con discapacidad.

El 26 de diciembre de 2007 se aprueba la Ley 49/2007, por la que se establece el régimen de infracciones y sanciones en materia de igualdad de oportunidades, no discriminación y accesibilidad universal de las personas con discapacidad. Esta ley ha venido a dotar de un instrumento jurídico a las personas con discapacidad para que puedan defenderse de las acciones discriminatorias que se puedan realizar contra ellas. Las sanciones para infracciones muy graves podrán llegar hasta un millón de euros. Ninguna de las leyes aprobadas

17 Kercher, 2007. 


\section{URBANI SMO}

ISSN 0717-5051

Revista de Urbanismo $N^{\circ} 28$ - Junio de 2013

http://revistas.uchile.cl/index.php/RU/index

Departamento de Urbanismo - FAU - Universidad de Chile

anteriormente en materia de discapacidad contenía régimen sancionador por lo que la vulneración de estas leyes ha resultado una práctica habitual.

El 17 de marzo de 2006 vio la luz el Real Decreto 314/2006 por el que se aprueba el Código Técnico de la Edificación sin regulación técnica en materia de accesibilidad, dado que todavía no se había aprobado el RD 505/2007 y por tanto no estaban reguladas las condiciones básicas de accesibilidad en la edificación. Esto no ocurre hasta el año 2010, cuando se aprueba el RD 173/2010, de 19 de febrero, por el que se modifica en el Código Técnico de la Edificación en materia de accesibilidad y no discriminación de las personas con discapacidad.

Como última disposición legal, es necesario detenernos en Ley 39/2006, de 14 de diciembre, de Promoción de la Autonomía Personal y Atención a las personas en situación de dependencia, la misma perdió la oportunidad de recoger el concepto de accesibilidad universal, cuando ya en su exposición de motivos reconocía sin reflejar el concepto de la importancia de actuar ante los cambios demográficos y sociales que están produciendo un incremento progresivo de la población en situación de dependencia. Por una parte, es necesario considerar el importante crecimiento de la población de más de 65 años, que se ha duplicado en los últimos 30 años, para pasar de 3,3 millones de personas en 1970 (un 9,7 por ciento de la población total) a más de 6,6 millones en 2000 (16,6 por ciento). A ello hay que añadir el fenómeno demográfico denominado "envejecimiento del envejecimiento", es decir, el aumento del colectivo de población con edad superior a 80 años, que se ha duplicado en sólo veinte años. Ambas cuestiones conforman una nueva realidad de la población mayor que conlleva problemas de dependencia en las últimas etapas de la vida para un colectivo de personas cada vez más amplio. A esta realidad, derivada del envejecimiento, debe añadirse la dependencia por razones de enfermedad y otras causas de discapacidad o limitación, que se ha incrementado en los últimos años por los cambios producidos en las tasas de supervivencia de determinadas enfermedades crónicas y alteraciones congénitas y, también, por las consecuencias derivadas de los índices de siniestralidad vial y laboral.

Además de todas las disposiciones legales anteriormente señaladas, existen otras herramientas para promover la accesibilidad. Entre ellas, conviene citar la Normalización y la Certificación; conceptos como la calidad, el medioambiente o la prevención de los riesgos laborales han tenido motores importantes en la normalización y la certificación, como lo demuestra el éxito de las normas ISO ${ }^{18}$ 9001, ISO 14001 o la especificación OHSAS $18001^{19}$. La Normalización y la Certificación se han revelado, además, como elementos de armonización entre las disposiciones de comunidades y países.

La Asociación Española de Normalización y Certificación (AENOR), por impulso de los agentes sociales, viene trabajando desde hace años en la normalización de la accesibilidad, aunque ha sido en el último lustro cuando estos trabajos se han intensificado de forma notable. Fruto de ellos son la elaboración de Normas UNE ${ }^{20}$ y la participación en las actividades de normalización

\footnotetext{
${ }^{18}$ Las normas ISO son elaboradas por la Organización Internacional para la Estandarización, especifican los requisitos para un Sistema de Gestión de la Calidad (SGC) que pueden utilizarse para su aplicación interna por las organizaciones, sin importar si el producto o servicio lo brinda una organización pública o empresa privada, cualquiera sea su tamaño, para su certificación o con fines contractuales.

19 OHSAS (Occupational Health and Safety Assessment Series). OHSAS 18001 (Occupational Health and Safety Assessment Series, Sistemas de Gestión de Salud y Seguridad Laboral) se refiere a una serie de especificaciones sobre la salud y seguridad en el trabajo, materializadas por BSI (British Standards Institution).

${ }^{20}$ Las normas UNE (Una Norma Española) son un conjunto de normas tecnológicas creadas por los comités técnicos de normalización (CTN), de los que forman parte todas las entidades y agentes implicados e interesados en los trabajos
} 
europea sobre el tema. Entre las Normas UNE elaboradas cabe hacer referencia a las siguientes:

- UNE 41500:2001 IN "Accesibilidad en la edificación y el urbanismo. Criterios generales de diseño".

- UNE 41501:2002 "Símbolo de accesibilidad para la movilidad. Reglas y grados de uso".

- UNE 41510:2001 "Accesibilidad en el urbanismo".

Muy recientemente se ha publicado la nueva norma UNE-ISO 21542:2012, de gran interés porque recoge toda la experiencia recopilada en una norma ISO muy completa y participada en su elaboración, esta norma de "Edificación. Accesibilidad del entorno construido" anula además a las normas UNE 41520, 41522 y 41523.

En el año 2001 se publicó la norma UNE 170.001 "Accesibilidad Global: Criterios para facilitar la accesibilidad al entorno" que consta de dos partes.

- Parte 1. Requisitos DALCO (Deambulación, Aprehensión, Localización y Comunicación).

- Parte 2. Sistema de gestión de la accesibilidad global.

Esta norma supone una ruptura de la línea seguida hasta la fecha por la normalización y presenta tres conceptos nuevos:

- Los requisitos DALCO: que plantean la La accesibilidad concebida desde la ineficacia del entorno para posibilitar las acciones básicas funcionales (criterios DALCO) y no desde el tipo de discapacidad.

- Establece un sistema de gestión de la accesibilidad y como consecuencia la mejora continua de ésta en un entorno construido.

- Es una norma certificable.

Requisitos DALCO: En su parte 1, la norma UNE 170.001 indica que "esta norma establece los requisitos que debe reunir un entorno (lugares, establecimientos, edificios e instalaciones) para que sus usuarios puedan superar las limitaciones de accesibilidad".

Los requisitos especificados en esta norma tienen por objeto "integrar las distintas necesidades de las personas en cualquier entorno construido para garantizar su uso de manera independiente y de la misma forma por parte de todos" y define los requisitos DALCO como "el conjunto de requisitos relativos a las acciones de deambulación, aprehensión, localización y comunicación que han de satisfacerse para garantizar la accesibilidad global de los entornos construidos".

El modelo de sistema de gestión de la accesibilidad global se basa en la estructura del Sistema de Gestión de la Calidad propuesto por la norma UNE-EN ISO 9001 y para su definición, sugiere examinar los procesos que el usuario de un entorno ha de seguir para su disfrute, a la luz de los requisitos DALCO. De esta forma se conocerán las necesidades de accesibilidad y una vez satisfechas se dispondrá de un entorno accesible.

El Sistema de Gestión de la Accesibilidad Global es certificable, es decir, puede ser evaluado por una entidad ajena e independiente, con el prestigio y la competencia necesarios que, de

del comité. Estos comités suelen estar formados por la Asociación Española de Normalización y Certificación, fabricantes, consumidores y usuarios, administración, laboratorios y centros de investigación 


\section{URBANI SMO}

acuerdo con los resultados, emite un certificado que evidencia que tal sistema cumple con los requisitos de la UNE $170.001^{21}$.

Esta norma fue revisada en 2007, sustituyendo el concepto de "Accesibilidad Global" por el de "Accesibilidad Universal". UNE 170001:2007

Si trasladamos toda esta normativa al ámbito autonómico, son las Comunidades Autónomas las que tienen potestad de legislar sobre la materia por lo que cada Comunidad Autónoma construye las Viviendas de Promoción Pública (VPP) de una manera diferente, aunque bien es cierto que todas las VPP tienen una serie de rasgos comunes. Todas las VPP tienen que estar regidas por el Decreto que regula el Plan Estatal de Vivienda y Rehabilitación 2009-2012.

\section{Metodología: Programa I ndividualizado de Accesibilidad}

La metodología ha consistido en un análisis en profundidad de distintas fuentes de información secundarias, destacando los portales telemáticos de la Unión Europea, Sistema para la Autonomía y Atención a la Dependencia (SAAD), Instituto de Mayores y Servicios Sociales (IMSERSO), Observatorio Estatal de la Discapacidad (OED); las bases de datos ProQuest y Dialnet; las distintas normativas relacionadas con el tema a nivel internacional, europeo, nacional y autonómicas; Directivas del Parlamento y del Consejo Europeo, Planes de Acción comunitaria; Leyes y Planes nacionales y autonómicos, y, por supuesto, la Ley 39/2006, de 14 de diciembre de Promoción de la Autonomía Personal y Atención a las personas en Situación de Dependencia, así como una extensa bibliografía nacional e internacional de autores de relevado prestigio en las materias relacionadas con el objeto de estudio.

El estudio comparado de las diversas legislaciones, investigaciones y respuestas autonómicas, ha dado como resultado la necesidad de intervenir proponiendo una herramienta generalizada e integral, destinada a este grupo de viviendas, y sobre todo teniendo presente las necesidades concretas de las personas que residen en ellas, diseñando un Programa Individualizado de Accesibilidad atendiendo a su diversidad funcional y tendente a su inclusión social, siempre bajo la filosofía del Design for all, ya que diseñando para toda la ciudadanía esa respuesta será perpetua para cualquier tipo de necesidad presente y futura.

Las situaciones de discapacidad/o dependencia evolucionan, obviamente, a lo largo de la vida de la persona. Sus necesidades de apoyo para desarrollar su plan de vida, también. Por eso, la atención centrada en la persona tiene un carácter procesual y exige que contenga la flexibilidad suficiente para realizar reajustes y adoptar decisiones y medidas ajustadas a cada momento. Este proceso continuo debe cursar en permanente diálogo (persona con discapacidad/ o en situación de dependencia-profesional) y entenderse, además de como un factor clave de la intervención, también como un método de aprendizaje recíproco y permanente para ambas partes de la relación ${ }^{22}$.

De los resultados obtenidos del trabajo de campo realizado en el Observatorio de la Accesibilidad Universal a los Municipios de España (Via Libre, 2011), se confirma que las actuaciones realizadas para mejorar la accesibilidad se han centrado en las necesidades de las personas con limitaciones en sus capacidades funcionales motóricas, especialmente usuarios de silla de ruedas, en relación a personas con limitaciones en sus capacidades sensoriales (visión, audición), orgánicas o cognitivas, se pone de manifiesto que se empieza a trabajar en ello pero

\footnotetext{
${ }^{21}$ Fontanals, 2006.

22 Martínez, 2010.
} 


\section{URBANI SMO}

con cierto retraso con respecto a los usuarios con discapacidad motora. Las actuaciones realizadas no han seguido un plan estratégico sino que se han realizado de forma puntual intentando resolver problemas parciales. Esto significa que no se ha realizado un análisis holístico de la situación en materia accesibilidad, por lo que no se han resuelto los problemas existentes teniendo en cuenta los principios del diseño para todos.

Por todo ello se propone la realización de un Protocolo de Rehabilitación con Criterios de Accesibilidad e Inclusión Social, humanizando la intervención y dotándola de empatía con las necesidades específicas del grupo de población al que atienden.

Este protocolo establecería directrices de información y actuación en políticas sociales de economía sostenible. El estudio ACCEPLAN ${ }^{23}$ concluye que se deberían mejorar los protocolos de valoración e intervención de los profesionales. Los informes que realizan deben estar estandarizados, bien estructurados y ser concisos y fácilmente informatizables e integrables en una base de datos u otro medio de tratamiento de información. Se deberían desarrollar formularios de valoración específicamente diseñados para incorporar e integrar las valoraciones de los distintos técnicos que puedan intervenir: trabajador social, arquitecto, arquitecto técnico, ingeniero, etc., haciendo más transparente y accesible la información entre ellos.

La decisión de intervenir sobre viviendas plurifamiliares de promoción pública anteriores a la entrada en vigor de los primeros decretos de accesibilidad se produce por las siguientes circunstancias:

- Son edificaciones en altura de entre 40 y 50 años que presentan un precario estado de conservación ya que:

o Fueron construidos con materiales de calidad básica y/o precarios servicios y medidas de confort.

o Están habitados por familias de rentas bajas, por lo que ha habido poca inversión en el mantenimiento y mejora.

- Son edificaciones que al haber sido construidas con anterioridad a los decretos de accesibilidad presentan numerosas barreras físicas tanto en zonas comunes como en el espacio habitable.

- Las personas que siguen ocupando estas viviendas son familias con rentas bajas y/o jubilados.

- Por lo general, la población que ocupan estas viviendas son longevas y podrían presentar importantes problemas motrices y/o cognitivos.

Son barrios y barriadas que, en algunos casos, ya ha sido objeto de políticas sociales de rehabilitación y recuperación de barrios en muchos aspectos como:

- Programas de integración social.

- Normalización de la propiedad.

- Programas de convivencia e inserción laboral.

\footnotetext{
23 La investigación ACCEPLAN fue financiada mediante subvención recibida de acuerdo con lo previsto en la Orden TAS/1051/2005, de 12 de abril (subvenciones para el Fomento de la Investigación de la Protección Social -FIPROS-) bajo el título "Adaptación de la vivienda en la población dependiente: necesidades, soluciones y costes".
} 


\section{URBANI SMO}

ISSN 0717-5051

http://revistas.uchile.cl/index.php/RU/index
Revista de Urbanismo $N^{\circ} 28$ - Junio de 2013

Departamento de Urbanismo - FAU - Universidad de Chile

- Mejora de infraestructuras y espacios de convivencia pública.

- Mejora de accesibilidad.

Sin embargo, se observa que en algunos casos dichos programas han quedado inconclusos o no han cubierto todos los frentes de actuación descritos por lo que no se consiguen los objetivos de cohesión e integración social.

Por sus características físicas, por su ubicación y por la situación social de sus inquilinos, este tipo edificaciones son susceptibles de integrarse en programas de rehabilitación en los términos establecidos por la Orden de 7 de julio de 2009, por la que se publica el texto integrado del Plan Concertado de Vivienda y Suelo 2008-2012, aprobado por el Decreto 395/2008, de 24 de junio, con las modificaciones introducidas por el Decreto 266/2009, de 9 de junio, en vigor actualmente. En muchos casos dichas intervenciones podrían hacerse extensibles a la zona, polígono y/o barrio donde estas se hallan insertas mediante la Declaración de zonas de actuación de infravivienda o de Rehabilitación Autonómica. Es decir, hablamos de políticas sociales y de economía sostenible en el más amplio significado del término regulado a través de la Ley 2/2011, de 4 de marzo, de Economía sostenible que establece en su ordenamiento las reformas estructurales necesarias para crear condiciones que favorezcan un desarrollo económico sostenible, entendiendo por economía sostenible un patrón de crecimiento que concilie el desarrollo económico, social y ambiental en una economía productiva y competitiva, que favorezca el empleo de calidad, la igualdad de oportunidades y la cohesión social, garantizando además el respeto ambiental y el uso racional de los recursos naturales.

El punto de Acceso a estas comunidades, y el punto de partida de estos protocolos se hallarían en la Ley 38/1999, de 5 de noviembre, de Ordenación de la Edificación, que establece que los propietarios y usuarios tienen la obligación de conservar en buen estado la edificación mediante un uso adecuado y mantenimiento. Así mismo, los documentos técnicos que penden de esta ley, es decir, el Código Técnico de la Edificación (CTE) (Real Decreto 314/2006), también recuerda la obligatoriedad de realizar un correcto mantenimiento del edificio por parte de propietarios y usuarios. Sin embargo, es el Decreto Ley 8/2011, publicado el 7 de julio de 2011, el que establece la obligatoriedad de realizar la Inspección Técnica de Edificios (ITE) a todos los inmuebles de más de 50 años de antigüedad (salvo que las Comunidades Autónomas fijen un límite menor), en los municipios de más de 25.000 habitantes. Este Decreto Ley entró en vigor definitivamente el pasado 8 de Julio de 2012.

\section{Resultados y Conclusiones: Efectos colaterales positivos de las intervenciones rehabilitadoras}

Analizar la accesibilidad desde el punto de vista de las situaciones de dependencia, no significa romper el concepto de accesibilidad universal, sino analizar e integrar en él, todas aquellas necesidades propias de este sector de la población, ya que esto será un requisito indispensable para una respuesta ajustada y adaptada.

No podemos olvidar que la intervención se realizará en el entorno más íntimo de la persona, la que supone la complejidad del hogar, que como unidad de análisis queda de manifiesto en palabras de Thomas Burch: 


\section{URBANI SMO}

ISSN 0717-5051

http://revistas.uchile.cl/index.php/RU/index
Revista de Urbanismo $N^{\circ} 28$ - Junio de 2013

Departamento de Urbanismo - FAU - Universidad de Chile

"El tamaño y la estructura del hogar pueden considerarse como funciones de tres grandes clases de factores afines: 1) la posibilidad; 2) la necesidad o la conveniencia;

3) la preferencia"24.

Es necesario destacar que las propuestas plateadas con anterioridad, darían lugar a resultados principales como serían la integración, el mantenimiento de las personas mayores y dependientes en sus domicilios el mayor tiempo posible, siguiendo así sus deseos intereses, viviendo incluidos y participando en igualdad en su comunidad y en su entorno, dando lugar así a una normalización en la respuesta que reciben por parte de la administración, sin generar una solución alejada de sus intereses y de sus necesidades. Facilitaría mucho el papel de las personas cuidadoras, en su mayoría mujeres (83\%) mayores de 56 años. El impacto socioeconómico estaría claramente justificado.

En lo referente a los resultados secundarios, es necesario destacar que pensar que los servicios sociales se puedan financiar únicamente con la aportación de las personas en situación de dependencia, es desconocer el coste de las prestaciones y sobre todo la situación económica de las personas en situación de dependencia.

En lo referente al coste medio por dependiente, para el caso de la gran dependencia R.D. $614 / 2007$ establece en su Anexo, que estima la aportación media de las administraciones públicas por beneficiario y mes en 1.014 euros para el nivel II de gran dependencia y en 774 euros para el nivel I de gran dependencia. En el caso de la dependencia severa se supone un gasto medio de 250 euros para el nivel II y de 200 euros para el nivel I, mientras que en el caso de la dependencia moderada se supone el coste prácticamente equivalente al gasto asociado a la prestación de servicios de teleasistencia, que se sitúa en 174 euros.

Para determinar la evolución futura de los gastos, Camacho, Rodríguez y Hernández ${ }^{25}$ toman como referencia la evolución mostrada por los precios de los servicios sociales durante los últimos años, estimando un crecimiento anual medio del coste por dependiente del 5,38\%.

Estimar cuál será el impacto financiero de la aplicación definitiva de la ley 39/2006, de 14 de diciembre, de Promoción de la Autonomía Personal y Atención a las Personas en Situación de Dependencia es una tarea considerablemente compleja, dado que entran en juego, no sólo las tendencias demográficas, sino también las condiciones de vida y de salud. Así lo han mostrado las notables disparidades que, aún bajo diferentes escenarios, se obtienen entre las estimaciones llevadas a cabo por diferentes organismos internacionales como la Organización para la Cooperación y el Desarrollo Económicos (OCDE) o las realizadas por la Comisión Europea, desde cifras que superan ampliamente el $1 \%$ del PIB (e incluso el $2 \%$ y el $3 \%$ en el caso de la OCDE), hasta valores situados por debajo de este nivel bajo las diferentes hipótesis analizada por la Comisión Europea. De distintas fuentes a la hora de estimar el volumen de población dependiente arroja un volumen de gasto en el sistema de atención a la dependencia en 2015 de unos 1347 millones de euros, gasto que se centra en gran medida en la atención a la gran dependencia. No obstante, que a estas cifras hemos de añadir el gasto actual en dependencia, que la OCDE cifra en el 0,2\% del PIB y la Comisión Europea en el 0,5\%.

En definitiva, dadas las ya reiteradas diferencias entre resultados, se hace imprescindible profundizar en el estudio del impacto financiero de la ley de dependencia, al objeto de

\footnotetext{
24 Burch 1976. p. 125.

${ }^{25}$ Camacho, Rodríguez y Hernández, 2008.
} 


\section{URBANI SMO}

ISSN 0717-5051

http://revistas.uchile.cl/index.php/RU/index
Revista de Urbanismo $N^{\circ} 28$ - Junio de 2013

Departamento de Urbanismo - FAU - Universidad de Chile

planificar con mayor exactitud las medidas de política económica necesarias para implementar de modo adecuado el sistema y sobre todo arbitrar medidas que faciliten la permanencia de las personas mayores o en situación de dependencia en su propio domicilio.

Por ello, se propone establecer un protocolo de información y actuación en materia de accesibilidad de carácter holístico toda vez que se realice la Inspección Técnica de Edificios que desde julio de 2012 es obligatoria en todo el territorio nacional, pero no para establecer únicamente un diagnóstico del edificio en cuanto al estado de conservación de edificio y eficiencia energética, sino diagnosticar además las condiciones de accesibilidad del inmueble y de sus viviendas así como las condiciones características y necesidades de sus moradores así como la situación socioeconómica de las familias. Es un buen momento para establecer estos parámetros en tanto que dado que esta legislación entró en vigor recientemente y dada la coyuntura económica que está atravesando el estado español, son muy pocos los municipios que han redactado la ordenanza en materia de Inspección Técnica de los Edificios (ITE) y muchos menos los que tienen puestos en marcha los programas de ITE al menos según los datos estadísticos del Observatorio ITE (Ministerio de Fomento) de donde se desprende que en muchas comunidades autónomas no se ha registrado ninguna ITE en el transcurso de 2012.

España cuenta por primera vez con un grupo de profesionales de diversos perfiles (arquitectos, ingenieros, aparejadores, informáticos, trabajadores sociales, psicólogos, enfermeros, etc.) capacitados en el marco del primer Máster Europeo de Accesibilidad Universal y Diseño para Todos, financiado por el Real Patronato de la Discapacidad, la Fundación Once, la Fundación Vodafone y la Fundación Repsol; que podrían dar respuesta a esta necesidad.

Las soluciones han de ser individualizadas, pero los profesionales que intervienen en la aplicación de la rehabilitación con criterios de accesibilidad deberían disponer de protocolos de intervención y recogida de información.

En este contexto, uno de los mayores cambios que este artículo propone alcanzar es el de variar las actitudes a todos los niveles, desde una manera de ver las adaptaciones como "bienestar" a otra que los vea como "inversión".

La insuficiente dotación de personal de los servicios, la falta de protocolos bien establecidos de diagnóstico, asesoramiento, visita, recopilación de información, valoración, etc., determina que los plazos de todo el proceso se alarguen de manera inadecuada y a veces desesperante para el usuario, situándolo así en una indefensión absoluta y en un riesgo para su seguridad personal.

Todas estas razones justificarían la realización de una intervención, ya no solo por el interés que representa en sí mismo, sino por dar respuesta a una población en situación de doble e incluso triple exclusión (en situación de pobreza/marginación, mayores, dependientes, feminización de la pobreza) y crear un grupo de apoyo de profesionales especialmente formados para dar respuesta a soluciones de entornos físicos y virtuales hostiles.

Las diferentes administraciones autonómicas, podría disponer de un protocolo de Rehabilitación, y de un grupo de profesionales para llevar a cabo intervenciones de rehabilitaciones accesibles con carácter integral y facilitaría la respuesta destinada a un grupo muy importante de ya demandantes o futuros demandantes tendrían cubiertas sus necesidades y podrían mantenerse en su domicilio una vez rehabilitado con criterios de accesibilidad (design thinking) pero aplicando innovación funcional y sobre todo el diseño emocional, situando en el centro de la intervención a las personas y a la usabilidad del producto diseñado. 


\section{URBANI SMO}

Este concepto aplica una visión mucho más holística al proceso de diseño intentando resolver problemas más complejos que los exclusivamente referentes a la forma y a la funcionalidad. Incorpora la resolución de problemas tales como la atención de necesidades de aquellos que se encuentran más alejados de la media como pueden ser los colectivos en riesgo de exclusión, personas en extrema pobreza, etc. ${ }^{26}$, añadiendo así lo que proponía Rob Imrie, dotando de un entendimiento mucho más sensibilizado y universal a los determinantes de la salud y de la calidad de vida 27 .

Todo ello con un apoyo más sencillo y viable para la administración, como es la teleasistencia domiciliaria, la ayuda a domicilio, o pequeñas intervenciones domóticas en el mismo, pero sobre todo con una atención más específica por parte de las promotoras inmobiliarias, ya que estas tienen un conocimiento limitado de las necesidades de las personas mayores y con discapacidad $^{28}$, siendo necesario que diseñen y promuevan los entornos construidos no pensando en usuario "medio", sino en un usuario que envejecerá, que podrá vivir solo, que necesitará apoyo de terceros o que pueda sufrir una discapacidad generada por una enfermedad degenerativa o sobrevenida. Un entorno que dé respuestas y que se adapte a los cambios naturales de la vida de una persona.

Es evidente que una estrategia de vivienda justa y eficaz para una sociedad que envejece, debiendo estar ésta plenamente integrada en todas las políticas de vivienda y planificación, ser flexible y con visión de futuro suficiente para tener en cuenta la riqueza relativa y la riqueza a través del mercado de la vivienda, ocuparse de ampliar las opciones y aumentando la calidad, independientemente de la tenencia, pero con un cuidado particular para aquellos que ahora viven en las peores viviendas, enfrentar y hacer frente a los riesgos de aislamiento social y riesgo evitable a través de la planificación económica y social, así como la mejora de la vivienda y la adaptación y reconocer que la discapacidad y el envejecimiento necesita políticas enfocadas a lo largo del curso de la vida.

\footnotetext{
${ }^{26}$ Brown and Wyatt, 2010.

27 Imrie, 2004.

${ }^{28}$ Imrie y Hall, 2001.
} 


\section{Bibliografía}

ACCEPLAN (2003). Por un Nuevo paradigma, el diseño para todos, para le plena igualdad de oportunidades. [En línea]. [Fecha de consulta: 3 mayo 2013]. Disponible en: http://www.ceapat.es/ceapat_01/centro_documental/diseno_todos/IM_034206

BROWN, Tim y WYATT, Jocelyn (2010). Design Thinking for Social I nnovation. Stanford Social Innovation Review. Vol. 8, Iss. 1, pp 31-35, Stanford University, Center for Social Innovation, Palo Alto. ISSN: 15427099

BURCH, Thomas (1976). Consideraciones sobre el análisis de la estructura del hogar y de la familia. En La familia como unidad de estudio demográfico (T. Burch, L. F. Lira \& V. F. Lopes, eds.) (pagina(s). 123140). San José (Costa Rica): CELADE

CAMACHO, José, RODRÍGUEZ, Mercedes y HERNÁNDEZ, Manuel (2008). El sistema de atención a la dependencia en España: Evaluación y comparación con otros países europeos. Cuadernos Geográficos. Vol. 42, pp 37-52. ISSN: 0210-5462

CERMI (2012). La transversalidad de género en las políticas públicas de discapacidad. [En línea]. [Fecha de consulta: 6 diciembre 2012]. Disponible en: http://www.cermi.es/esES/Biblioteca/Paginas/I nicio.aspx?TSMEI dPub $=288$

COMMUNITIES AND LOCAL GOVERNMENT (2008). Lifetime Homes, Lifetime Neighbourhoods. A National Strategy for Housing in an Ageing Society. [En línea]. [Fecha de consulta: 9 mayo 2013]. Disponible en http://www.imsersomayores.csic.es/documentos/documentos/dh-lifetime-01.pdf

DE ASIS, Rafael (2007). Reflexiones en torno a la Ley de protección de la autonomía personal y atención a las personas en situación de Dependencia. [En línea]. [Fecha de consulta: 5 mayo 2013]. Disponible en: http: //universitas.idhbc.es/n05/05-02.pdf

EIDD. European Institute for Design and Disability (2004). The EIDD Stockholm Declaration 2004. [En línea]. 2004. [Fecha de consulta: 8 diciembre 2012]. Disponible en: http://www.designforalleurope.org/Design-for-All/EIDD-Documents/Stockholm Declaration/

Fontanals, Jaime. Certificado en Accesibilidad, garantía de Calidad. En I Congreso Internacional de Turismo para Todos. [En línea]. 2004. [Fecha de consulta: 25 noviembre 2012]. Disponible en: http://www. discapnet.es/Castellano/areastematicas/Accesibilidad/turismoparatodos/ES/congreso2004/Pa ginas/default. aspx

Imrie, Rob. Demystifying disability: a review of the International Classification of Functioning, Disability and Health. Sociology of Health \& Illness Vol. 26 No. 3. 2004 ISSN 0141-9889, pp. 287-305.

Imrie, Rob y Hall Peter. An Exploration of Disability and the Development Process. [En línea]. 2001. [Fecha de consulta: 4 mayo 2013]. Disponible en: http://usj. sagepub.com/content/38/2/333. full. pdf

IMSERSO. Instituto de Migraciones y Servicios Sociales. Plan Nacional de Accesibilidad 2004-2012. Ministerio de Trabajo y Asuntos Sociales. [En línea]. 2003. [Fecha de consulta: 29 noviembre 2012]. Disponible en: http://sid. usal.es/libros/discapacidad/12610/8-1/i-plan-nacional-de-accesibilidad-20042012.aspx

IMSERSO. Instituto de Migraciones y Servicios Sociales. Libro Verde de la Accesibilidad. Ministerio de Trabajo y Asuntos Sociales. [En línea]. 2002. [Fecha de consulta: 29 noviembre 2012]. Disponible en: http://www.ceapat.es/ceapat_01/centro_documental/accesibilidad/accesibilidad_entorno/IM_034193

IMSERSO. Instituto de Migraciones y Servicios Sociales. Concepto europeo de accesibilidad. [En línea]. 1996. [Fecha de consulta: 8 enero 2013]. Disponible en: http://www.ceapat.es/ceapat_01/centro_documental/accesibilidad/accesibilidad_entorno/IM_032953 


\section{REVISTA DE}

\section{URBANI SMO}

ISSN 0717-5051

Revista de Urbanismo N²8 - Junio de 2013

http://revistas.uchile.cl/index.php/RU/index

Departamento de Urbanismo - FAU - Universidad de Chile

INSTITUTO ARAGONÉS DE EMPLEO. Discapacidad y Empleo. [En línea]. 2005. [Fecha de consulta: 1 mayo 2013]. Disponible

en:

http://www.aragon.es/estaticos/I mportFiles/07/docs/Areas\%20Genericas/Publicaciones/DISCAPACIDAD_

EMPLEO.pdf

Juncà, José Antonio. Reflexiones en torno a la Accesibilidad Universal. De cómo transitar de las ideas a las realidades. En A fondo. [En línea]. 2010. [Fecha de consulta: 1 mayo 2013]. Disponible en: http: // riberdis.cedd. net/xmlui/ bitstream/handle/11181/3186/186748. pdf? sequence $=1$

Kercher, Pete. Interview with Pete Kercher. [En línea]. 2007. [Fecha de consulta: 24 noviembre 2012]. Disponible en: http://www.designforalleurope.org/Design-for-All/Articles/ Interview-with-Pete-Kercher-inDelo-magazine/.

Palacios, Agustina. El modelo social de discapacidad: orígenes, caracterización y plasmación en la Convención Internacional sobre los Derechos de las Personas con Discapacidad. [En línea]. 2008. [Fecha de consulta: 5 mayo 2013]. Disponible en: http://www.convenciondiscapacidad.es/Publicaciones_new/6_El\% 20modelo\% 20social\% 20de\% 20discapac idad.pdf

Martínez, Teresa. Centros de atención diurna para personas mayores. Atención a las situaciones de fragilidad y dependencia. Madrid, Médica Panamericana. 2010. 282 p. ISBN 978-849-83-5284-9

Montorio, Ignacio. Intervenciones psicológicas en la prevención y atención a la dependencia. Revista Intervención Psicosocial, 16, 43-54. Madrid. 2007. ISSN: 1132-0559

Oliver, Mike. The politics of disablement. Londres: Macmillan. 1990. ISBN 033-3432-924

Rioux, Liliane. The well-being of aging people living in their own homes. Journal of Environment Psychology 25, 231-243. University of Georgia, USA. 2005. ISSN 2171-1976

Rodríguez, Pilar. La atención integral centrada en la persona. [En línea]. 2010. [Fecha de consulta: 28 noviembre 2012]. Disponible en: http://www.imsersomayores.csic.es/documentos/documentos/pilaratencion-01.pdf

Shakespeare, Tom. Disability Rights and Wrong. London. Routledge.2006.

Thomas, Carol. How is disability understood? An examination of sociological approaches. Disability and Society. Vol. 19, No. 6, October 2004. ISSN 0968-7599.

Vía Libre. Observatorio de la Accesibilidad Universal en los Municipios de España. [En línea]. 2011. [Fecha de consulta: 3 octubre 2012]. Disponible http: //sid. usal. es/idocs/F8/FDO26092/Observatorio\% 20accesibilidad\%20municipios\% 202011.pdf 